D) Check for updates

Cite this: Food Funct., 2020, 11, 5333

\section{A comprehensive investigation of gluten free bread dough rheology, proving and baking performance and bread qualities by response surface design and principal component analysis $\uparrow$}

\author{
Yi Ren, (D) *a Bruce R. Linter, ${ }^{b}$ Robert Linforth ${ }^{a}$ and Tim J. Foster ${ }^{* a}$
}

\begin{abstract}
Contribution of methylcellulose (MC), psyllium seed husk powder (PSY), and water addition level to gluten free bread quality and correlations between dough rheological properties and bread qualities were investigated by response surface design and principal component analysis. The generalised Maxwell model was applied to estimate the relaxation frequency of gluten free doughs. The addition of PSY has a complex influence on pasting viscosity at high temperature and an additional peak was observed. MC significantly influenced dough extensibility and work of adhesion, which are good predictors of bread volume and textural properties. Other rheological responses are less significantly correlated to specific volume, but they are sensitive to formulation variations, reflect dough structures and stability, related to proving behaviours, and correlated to loaf concavity. An inappropriate combination of water and hydrocolloids might lead to problems such as low stability of doughs, overexpansion, and weak crumb structure at high water addition levels, or, in contrast, high rigidity of dough, a trap of excessive air during mixing, and restrained gas cell expansion with high hydrocolloid addition and low water addition.
\end{abstract}

Received 13th January 2020, Accepted 21st May 2020

DOI: $10.1039 / \mathrm{dOfo} 00115 \mathrm{e}$ rsc.li/food-function qualities. ${ }^{3,4}$ For the purpose of dealing with gluten intolerance and meeting the "free-from" trend, the production and quality improvement of gluten free bread are becoming a new favourite of both manufacturers and researchers. However, it is still a challenge to create products with acceptable sensory attributes.

Currently, several methodologies have been developed for gluten-free bread manufacturing, which can be categorised as the application of alternative flour blends, enzyme treatment and sourdough application, and other treatments, among which, the application of alternative flour blends is the most investigated. Alternative flour is mostly a blend of one or more types of starch and/or starch-based flours and, in most cases, hydrocolloids. A preferable alternative flour is rice flour, because of its bland taste, white colour, low sodium content, easily digestive carbohydrates and hypoallergenic properties. ${ }^{5}$ Hydrocolloids are the other type of ingredients in the alternative flour blends, usually with a small amount of addition, but, significant functionality. One of the early studies on rice bread was conducted by Nishita $e t$ al. ${ }^{6}$ who investigated the addition of different kinds of hydrocolloids, dough conditioners and lipids. They reported that hydroxypropyl methylcellulose (HPMC) and refined vegetable oils gave better loaves in the absence of dough conditioners and surfactants. Haque and Morris $^{7}$ also investigated rice flour based bread with the addition of HPMC and psyllium seed husk powder and obtained higher aeration than most commercial wheat bread. 
Most research on gluten free bread includes hydrocolloid addition to enhance the structure building, improve mouthfeel, increase shelf-life and increase the overall acceptability. ${ }^{8,9}$ The most commonly used hydrocolloid in a gluten-free bread formula is HPMC. ${ }^{10}$ Compared to HPMC, methylcellulose (MC) is less studied and applied in gluten free bread. It forms thermoreversible gels at a lower temperature $\left(50-55{ }^{\circ} \mathrm{C}\right)$ than HPMC with a higher gel strength. The gelling property at high temperature is similar to gluten, which denatures and forms a stable network during heating and contributes to the final texture of bread crumb. ${ }^{7}$ Recently, the seed husk of psyllium (Plantago ovata Forsk), also known as ispaghula or isabgol, is widely applied in gluten free bread production. The mucilage of hydrated psyllium seed husk is mainly composed of heteroxylans, which are mainly substituted, by arabinose and xylose. As a natural source of dietary fibre, it has been traditionally used in medical treatment in some countries and it is proven that psyllium has the ability to lower cholesterol levels, be used as laxative, and improve insulin sensitivity. ${ }^{11-13}$ Although the mucilage of psyllium seed husk does not dissolve in water, its dispersion shows a 'weak gel' property which is similar to xanthan. ${ }^{14}$ In addition, water content is critical in bakery products which influences quality, texture, taste, smell, volume, flavours and mouthfeel ${ }^{15}$ as its mobility and distribution influence dough rheology and baking performance. ${ }^{16}$ It has been emphasised that an applicable water addition level is critical in gluten free bread formulation. , $^{17}$

Gluten free doughs and bread have been widely investigated. ${ }^{17-23}$ Dough rheology can be an indicator of the quality of end products. ${ }^{24}$ However, dough characterisation, crumb structure and dough-crumb transformation can be complicated as it involves dough stability, gas trapping ability and water distribution in doughs, which further influences starch gelatinisation, structure transformation, and crumb formation during baking. However, the information and properties investigated are limited in most studies.

This study investigated the roles of MC and psyllium seed husk powder (PSY) at different water addition levels in gluten free doughs and bread by response surface design (RSD). An effort has been made to generate a comprehensive characterisation, which leads to a large amount of data. Bratchell ${ }^{25}$ firstly applied principal component analysis (PCA) to RSD data which was later followed by Ribeiro et $a l^{26}$ and Das Purkayastha et $a .^{27}$ A combination of PCA and RSD was applied in this study to reduce the number of data dimensions and study the correlations between responses. To our best knowledge, this is the first attempt to combining PCA with RSD to investigate the addition of hydrocolloids in gluten free doughs and bread to generate a more comprehensive characterisation of the whole bread making process and bread quality.

\section{Materials and methods}

\subsection{Materials}

Rice flour (11.08\% moisture, $7.23 \%$ protein, $0.42 \%$ ash, $2.8 \%$ lipid, and $28.8 \%$ amylose as measured in a previous study ${ }^{28}$ ) was purchased from Doves Farm. Allinson Easy Bake Yeast, sugar, sunflower oil, and salt were purchased from local supermarkets. Methylcellulose (Methocel ${ }^{\circledR}$ A4M) were purchased from The DOW Chemical Company (Bomlitz, Germany). Psyllium seed husk powder (Vitacel ${ }^{\circledR}$ ) (7.23\% moisture, 3.40\% protein, $2.89 \%$ ash, and $3.30 \%$ lipid as measured in a previous study ${ }^{28}$ ) was kindly donated by the JRS (J. Rettenmaier \& Söhne Group, Rosenberg, Germany).

\subsection{Formulation and dough preparation}

The basic formulation included $100 \mathrm{~g}$ of rice flour, $5 \mathrm{~g}$ of sugar, $2 \mathrm{~g}$ of salt, $1.5 \mathrm{~g}$ of yeast, and $5 \mathrm{~g}$ of sunflower oil. The addition levels of water, MC and PSY are shown in Table 1 according to IV-optimal design with the total hydrocolloid addition level no more than $3.5 \mathrm{~g}$ per $100 \mathrm{~g}$ of rice flour. Doughs were prepared based on $200 \mathrm{~g}$ of rice flour per batch by a stand mixer (Kenwood, UK) equipped with a CHEF flexible beater (AT501, Kenwood, Havant, UK). Dry ingredients were mixed thoroughly and then mixed with water and oil for $7 \mathrm{~min}$ at speed 1.

\subsection{Dough evaluation}

2.3.1. Fundamental rheological measurements. Dough sample preparation is described in the previous section (2.2) excluding the addition of yeast. Samples were sealed in plastic containers and rested at room temperature for $1 \mathrm{~h}$ before experiments. Controlled shear stress tests and small amplitude oscillatory tests were performed using an MRC 301 rheometer (Anton Paar, Austria) with serrated parallel plate geometry (PP25/P2-SN15766, Anton Paar) and a measuring gap was $2 \mathrm{~mm}$. The temperature was controlled by a Peltier system which was connected to a water bath (R1, Grant, Shepreth). Low viscosity mineral oil (Sigma, USA) was applied to the edge of the samples to prevent drying. Doughs were loaded and

Table 1 Addition levels of water, MC and PSY per $100 \mathrm{~g}$ of rice flour according to IV-optimal design

\begin{tabular}{llll}
\hline Run & Water & MC & PSY \\
\hline 1 & 130 & 0.2 & 0 \\
2 & 130 & 2 & 1 \\
3 & 120 & 0 & 1 \\
4 & 110 & 0 & 2 \\
5 & 110 & 1 & 0.7 \\
6 & 120 & 0 & 1 \\
7 & 120 & 1 & 1 \\
8 & 125 & 1.2 & 0.3 \\
9 & 110 & 2 & 1.5 \\
10 & 110 & 1 & 0.7 \\
11 & 110 & 0 & 0 \\
12 & 125 & 1.2 & 0.3 \\
13 & 110 & 2 & 0.5 \\
14 & 120 & 0.5 & 2 \\
15 & 115 & 2 & 0 \\
16 & 122 & 1.5 & 2 \\
17 & 130 & 0 & 2 \\
18 & 112 & 1.2 & 2 \\
19 & 118 & 1.8 & 1 \\
20 & 128 & 0.8 & 1.3
\end{tabular}


rested at $30{ }^{\circ} \mathrm{C}$ for $500 \mathrm{~s}$ before measurement. Controlled shear stress tests were performed to obtain the yield point (yield stress and strain) with the stress increasing logarithmically from 0.03 to $30000 \mathrm{~Pa}$ over $18.5 \mathrm{~min}$. The yield point was determined by defining an upward bending point with $1 \%$ bandwidth using yield stress II in Rheoplus 3.4. Frequency sweep tests were performed in a logarithmic decrease manner with a strain of $0.02 \%$ which is in the linear viscoelastic region. The frequency dependences of storage moduli $G^{\prime}$ and complex viscosity $\eta^{*}$ in the middle frequency range $(0.881$ to $40.9 \mathrm{rad} \mathrm{s}^{-1}$ ) were represented by the slopes of $\log G^{\prime}$ versus $\log \omega$ and $\log \eta^{*}$ versus $\log \omega$ respectively. The obtained mechanical spectra were fitted to the generalised Maxwell model. The dynamic moduli $G^{\prime}(\omega)$ and $G^{\prime \prime}(\omega)$ in small amplitude oscillatory experiments can be estimated and calculated from angular frequency $(\omega)$ by eqn (1) and (2). ${ }^{29-31}$ The fitting was performed by varying individual relaxation moduli $G_{\mathrm{i}}$ minimising the sum of squared differences between calculated $G^{\prime}(\omega), G^{\prime \prime}(\omega)$ and experimentally obtained $G^{\prime}, G^{\prime \prime}$ with arbitrarily decided $\lambda_{\mathrm{i}}$ as shown in Table 2. $G_{\mathrm{e}}$ represents a pure elastic component in the model. All fundamental rheological measurements were repeated at least twice.

$$
\begin{gathered}
G^{\prime}(\omega)=\sum_{i} G_{\mathrm{i}} \frac{\omega^{2} \lambda_{\mathrm{i}}{ }^{2}}{1+\omega^{2} \lambda_{\mathrm{i}}{ }^{2}}+G_{\mathrm{e}} \\
G(\omega)=\sum_{i} G_{\mathrm{i}} \frac{\omega \lambda_{\mathrm{i}}}{1+\omega^{2} \lambda_{\mathrm{i}}^{2}}
\end{gathered}
$$

2.3.2. Empirical rheological measurements. Doughs were prepared as described in section 2.2 without the addition of yeast. Samples were sealed in plastic containers and rested at room temperature for $1 \mathrm{~h}$ before analysis using a backward extrusion rig and SMS/Chen-Hoseney dough stickiness rig (Stable Micro Systems, UK) on a TA-XT plus texture analyser
(Stable Micro systems, Surrey, UK) equipped with a $5 \mathrm{~kg}$ loading cell. In the backward extrusion test, doughs were loaded into a container (diameter of $42 \mathrm{~mm}$ ) to a height of $36 \mathrm{~mm}$ avoiding big air pockets. A disc with a diameter of $30 \mathrm{~mm}$ and thickness of $5 \mathrm{~mm}$ centrally extruded into the dough by $22.5 \mathrm{~mm}$ and then returned to the height of $120 \mathrm{~mm}$ from the base at a speed of $1 \mathrm{~mm} \mathrm{~s}^{-1}$. The start of data recording was triggered by a load of $5 \mathrm{~g}$. The positive peak force during the downward stroke and negative peak during the upward stroke were marked as dough firmness and cohesiveness respectively. The positive and negative areas were calculated as dough consistency and index of viscosity respectively. Dough extensibility was the returning distance before the force reached a constant negative value. For the stickiness test, doughs were loaded into a SMS/Chen-Hoseney Dough Stickiness Cell (A/DSC) and a uniform surface was generated. ${ }^{32}$ The surface was compressed at a speed of $0.5 \mathrm{~mm} \mathrm{~s}^{-1}$ by a $25 \mathrm{~mm}$ aluminium cylinder probe $(\mathrm{P} / 25)$ with a target load of $40 \mathrm{~g}$ for $0.1 \mathrm{~s}$, after which the probe withdrew at $10 \mathrm{~mm} \mathrm{~s}^{-1}$. The maximum force, area, and travel distance during probe withdrawing indicated dough stickiness, work of adhesion, and strength individually. Five replicates of each measurement were performed.

2.3.3. Pasting properties of flour blends. The flour blends were prepared by mixing rice flour with MC or/and PSY in the same ratio as shown in Table 1. Sugar, salt, yeast, and oil were excluded. The pasting properties of flour blends were characterised by Rapid Visco Analyser (RVA) (Newport Scientific Pty. Ltd, Warriewood, New South Wales, Australia) equipped with a water bath (Thermo scientific C10, Karlsruhe, Germany). The flour blends containing $2.5 \mathrm{~g}$ of rice flour and various amounts of MC and/or PSY were dispersed in $24 \mathrm{ml}$ of RO water. After initial $60 \mathrm{~s}$ mixing at a high shear rate $(960 \mathrm{rpm})$ and another $60 \mathrm{~s}$ mixing at $160 \mathrm{rpm}$ at $25^{\circ} \mathrm{C}$, the temperature increased to $95{ }^{\circ} \mathrm{C}$ in $350 \mathrm{~s}$, held at $95{ }^{\circ} \mathrm{C}$ for $150 \mathrm{~s}$, and

\begin{tabular}{|c|c|c|c|c|c|c|c|c|c|c|c|}
\hline $\begin{array}{l}\text { Individual relaxation } \\
\text { time } \lambda_{\mathrm{i}}(\mathrm{s})\end{array}$ & 0.0001 & 0.001 & 0.01 & 0.1 & 1 & 10 & 100 & 1000 & 10000 & 100000 & $R^{2}$ \\
\hline Run 1 & 27081 & 0 & 0 & 31 & 205 & 587 & 0 & 0 & 0.008 & 0.001 & 0.725 \\
\hline Run 2 & 0 & 9375 & 3944 & 1724 & 842 & 1403 & 1404 & 0.203 & 0.010 & 0.001 & 0.985 \\
\hline Run 3 & 12853 & 0 & 2128 & 436 & 541 & 592 & 1760 & 0.203 & 0.010 & 0.001 & 0.888 \\
\hline Run 4 & 0 & 9301 & 4038 & 3044 & 2201 & 1948 & 6658 & 0.213 & 0.012 & 0.001 & 0.994 \\
\hline Run 5 & 0 & 4369 & 6429 & 1329 & 1521 & 2584 & 2857 & 0.204 & 0.010 & 0.001 & 0.895 \\
\hline Run 6 & 0 & 2326 & 1472 & 491 & 451 & 604 & 1471 & 0.219 & 0.014 & 0.001 & 0.930 \\
\hline Run 7 & 0 & 2203 & 4455 & 570 & 1032 & 0 & 2327 & 0.204 & 0.010 & 0.001 & 0.862 \\
\hline Run 8 & 21548 & 0 & 2469 & 434 & 0 & 1288 & 0 & 0.000 & 0.010 & 0.001 & 0.881 \\
\hline Run 9 & 180032 & 0 & 6957 & 4510 & 2281 & 5825 & 2733 & 0.202 & 0.010 & 0.001 & 0.998 \\
\hline Run 10 & 0 & 5995 & 4750 & 1232 & 1237 & 1782 & 2503 & 0.205 & 0.010 & 0.001 & 0.928 \\
\hline Run 11 & 0 & 0 & 1869 & 0 & 72 & 1100 & 0 & 0.000 & 0.009 & 0.033 & 0.629 \\
\hline Run 12 & 0 & 3851 & 2660 & 363 & 458 & 0 & 997 & 0.202 & 0.010 & 0.001 & 0.889 \\
\hline Run 13 & 0 & 15699 & 5603 & 3200 & 1362 & 3597 & 1188 & 0.202 & 0.010 & 0.001 & 0.997 \\
\hline Run 14 & 125673 & 0 & 554 & 2252 & 1083 & 3477 & 1949 & 0.203 & 0.010 & 0.001 & 0.897 \\
\hline Run 15 & 0 & 12560 & 4031 & 2204 & 903 & 3223 & 0 & 0.201 & 0.010 & 0.001 & 0.996 \\
\hline Run 16 & 133958 & 0 & 3586 & 2635 & 1193 & 3709 & 1092 & 0.203 & 0.010 & 0.001 & 0.996 \\
\hline Run 17 & 67997 & 0 & 0 & 1100 & 560 & 1726 & 1051 & 0.202 & 0.010 & 0.001 & 0.712 \\
\hline Run 18 & 0 & 12918 & 6920 & 3305 & 2575 & 3437 & 4957 & 0.206 & 0.011 & 0.001 & 0.982 \\
\hline Run 19 & 0 & 9833 & 5409 & 2011 & 1280 & 833 & 2569 & 0.206 & 0.010 & 0.001 & 0.974 \\
\hline Run 20 & 0 & 4678 & 2722 & 695 & 736 & 860 & 1343 & 0.202 & 0.010 & 0.001 & 0.917 \\
\hline
\end{tabular}

Table 2 Individual relaxation time and relaxation moduli for generalised Maxwell model fitting 
decreased to $25^{\circ} \mathrm{C}$ in $350 \mathrm{~s}$. Data were collected and peak 2, peak 3 , trough 1 , trough 2 , trough total, breakdown, final viscosity, setback, peak 2 time, peak 3 time, peak 2 temp, peak 3 temp, and pasting temp were analysed by the software, Thermocline. All measurements were performed in triplicate.

\subsection{Baking tests}

Gluten free doughs were prepared according to section 2.2 then 200 to $210 \mathrm{~g}$ portion of dough were loaded in a baking pan with a dimension of $7.5 \times 7.5 \times 10 \mathrm{~cm}$. Two doughs were generated per batch of mixing. The baking pan was shaken to avoid big air pockets and the doughs were covered with cling film and proved in an incubator (Binder, Tuttlingen, Germany) at $30^{\circ} \mathrm{C}$ for $85 \mathrm{~min}$. Baking was perfomed at $230^{\circ} \mathrm{C}$ for $40 \mathrm{~min}$ in a deck oven (Tom Chandley, Manchester, UK). The loaves were Then cooled on a rack at ambient condition for $1 \mathrm{~h}$ before further operations.

2.4.1. Basic analysis and calculations. The proving behaviour was monitored by loading approximately $10 \mathrm{ml}$ of dough sample in a measuring cylinder which was incubated at $30^{\circ} \mathrm{C}$ for 85 min during which the volume was recorded. Volume increase max, volume increase final, maximum rate and maximum rate time were calculated. After cooling, the loaves were weighed and loaf volumes were measured by rapeseed displacement. The baking loss was calculated by eqn (3). Specific volume was the ratio of loaf volume to loaf weight. A piece of bread crumb was cut from the centre of the loaves and the moisture content was measured.

$$
\begin{aligned}
& \text { baking loss = } \\
& \frac{\text { dough weight before proving }- \text { loaf weight after cooling }}{\text { dough weight before proving }}
\end{aligned}
$$

The loaves were cut into $1.25 \mathrm{~cm}$ thick slices and the surface of the middle slice was scanned by C-Cell (Calibre Control International LTD, Warrington, UK). The obtained images were analysed using the equipped software and twentythree parameters including slice area, area of cells, area of voids, cell diameter, wall thickness and top concavity were obtained.

2.4.2. Bread textural evaluation. Texture profile analysis (TPA) was performed on a TA-XT plus texture analyser (Stable Micro systems) equipped with $30 \mathrm{~kg}$ loading cell where the sample was compressed to $65 \%$ of its original height twice by a $100 \mathrm{~mm}$ plate at a speed of $1 \mathrm{~mm} \mathrm{~s}^{-1}$ with $5 \mathrm{~s}$ between two compressions. The bread crumb was cut from the centre of the four middle loaf slices in a cylinder shape with a diameter of $3 \mathrm{~cm}$. Two cylinder pieces were stacked together and reached a height of $2.5 \mathrm{~cm}$. Hardness, springiness, cohesiveness, gumminess, chewiness, and resilience were calculated from the TPA profiles. The measurements were repeated for four times for each composition.

\subsection{Experiment design and analysis}

The experiment was designed using IV-optimal design (RSD) by Design expert 9 based on three factors, which were addition levels of PSY, MC and water, and this involved 20 runs (Table 1). The lack of fit of the models was evaluated by 6 runs and 4 runs were repeated twice to evaluate error values. Both doughs and loaves were assessed and 74 responses were obtained to analyse the influence of the three factors and correlations between these responses. The regression model of each responses was calculated and analysed by Design expert 9 (Stat-Ease Inc., Minneapolis, MN, USA) using analysis of variance (ANOVA) at a significance level of $p<0.05, p<0.01, p<$ 0.001 , or $p<0.0001$ and $R^{2}$ was identified. PCA was performed using Minitab 17.2.1 (Minitab, LLC).

\section{Results and discussion}

\subsection{Fundamental rheological analysis of dough properties}

The majority of the dough and porous crumb structure is developed during proving. The deformation rates during proving are at low magnitude in a range from $10^{-4}$ to $10^{-3} \mathrm{~s}^{-1}$ as reported by Babin et al. ${ }^{33}$ and Grenier et al. ${ }^{34}$ Therefore, it is beneficial to understand the rheological behaviour of doughs at a longer timescale including their relaxation time. Wyss et $a l .{ }^{35}$ published Strain-Rate Frequency Superposition (SRFS) for metastable soft materials by shear rate controlled frequency sweep tests which, therefore, involve large amplitude oscillation when the shear rate is relatively high and angular frequency is low. However, this technique might generate controversial results due to nonlinear contributions. ${ }^{36}$ Additionally, most gluten free doughs in this study did not show a pronounced structural relaxation peak in the measurable frequency range, possibly due to low stabilities and short linearity, which influences the generation of master curves. Therefore, it is problematic to apply SRFS to gluten free rice doughs.

Another method is the generalised Maxwell model. A single Maxwell model is composed of a serially connected Newtonian dashpot characterised by viscous response $\eta$ and a Hookean spring characterised by elastic response $G$, which produce an overall relaxation time $\lambda$. The generalised Maxwell model consists of several single Maxwell elements connected in parallel where each element is characterised by $G_{\mathrm{i}}$ and $\lambda_{\mathrm{i}}$. A single spring can be included in the generalised Maxwell model characterised by $G_{\mathrm{e}}$. The model fitting in this study led to 21 parameters including 10 predefined $\lambda_{\mathrm{i}}$ for each Maxwell element and 10 corresponding $G_{\mathrm{i}}$ as shown in Table 2, and $G_{\mathrm{e}}$. $G_{\mathrm{e}}$ for all the samples was either zero or close to zero suggesting an insignificant contribution to the overall viscoelasticity and, therefore, was not listed in Table 2.

Four typical mechanical spectra and fittings to the generalised Maxwell model (run 1, run 2, run 6, and run 11) are shown in Fig. 1 showing the experimentally obtained storage $\left(G^{\prime}\right)$ and loss moduli $\left(G^{\prime \prime}\right)$, and calculated moduli $\left(G^{\prime}(\omega)\right.$ and $G^{\prime \prime}$ $(\omega))$. All four doughs are solid-like as $G^{\prime}$ is higher than $G^{\prime \prime}$ although they are different in the frequency dependence of moduli. The generalised Maxwell model fits the experimental data with a satisfactory $R^{2}$ (Table 2), however the fitting of $G^{\prime \prime}$ is 


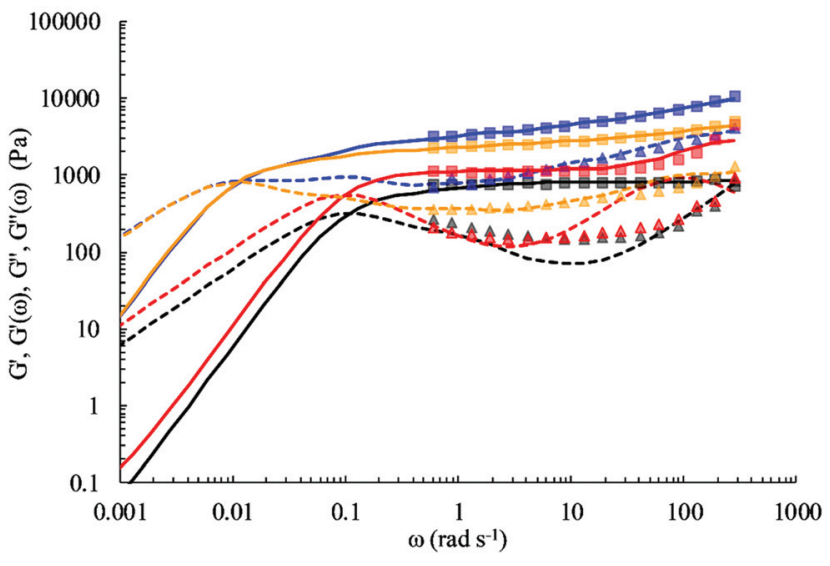

Fig. 1 Mechanical spectra and curves calculated from the generalised Maxwell models of run 1 (black), run 2 (blue), run 6 (yellow), and run 11 (red). Experimentally measured storage moduli $\left(G^{\prime}\right)$ and loss moduli ( $\left.G^{\prime \prime}\right)$ are shown as square and triangle symbols respectively and calculated $G^{\prime}$ $(\omega)$ and $G^{\prime \prime}(\omega)$ are presented as solid lines and dashed lines singly. not always accurate over the angular frequency range applied in the experiment as seen in Fig. 1 (run 1, 6 and 11). The lack of fit mainly occurred at the higher angular frequency range (approx. 20 to $120 \mathrm{rad} \mathrm{s}^{-1}$ ) as seen in run 6 in the same figure. There can be additional deviations from experimentally measured $G^{\prime \prime}$ at the lower frequency range at around $2.5 \mathrm{rad}$ $\mathrm{s}^{-1}$ (run 11). Run 1 was the only one in all 20 runs showing lack of fit in the medium angular frequency range. Therefore, for each run in RSD, the storage and loss moduli, complex viscosity, and $\tan \delta$ at two angular frequencies (1.29 and $60 \mathrm{rad}$ $\mathrm{s}^{-1}: G_{1.29}^{\prime}, G_{60}^{\prime}, G^{\prime \prime}{ }_{1.29}, G^{\prime \prime}{ }_{60}, \eta_{1.29}^{*}, \eta_{60}^{*}, \tan \delta_{1.29}$, and $\tan \delta_{60}$, slopes of $\log G^{\prime}$ versus $\log \omega$ and $\log \eta^{*}$ versus $\log \omega$, predicted relaxation frequency $\left(\omega_{\text {rel }}\right)$, with corresponding moduli $\left(G_{\text {rel }}^{\prime}\right)$ and complex viscosity $\left(\eta_{\text {rel }}^{*}\right)$, and $R$ square values of the generalised Maxwell model $\left(R_{\mathrm{M}}^{2}\right)$ are involved in further analysis.

All three factors had significant effects on moduli and complex viscosity at both high and low angular frequencies (1.29 and $60 \mathrm{rad} \mathrm{s}^{-1}$ ) with $P<0.0001$ (Table 3), where the coefficients of each term in the regression models for $G_{60}^{\prime}$,

Table 3 Coefficients of model terms for response surfaces

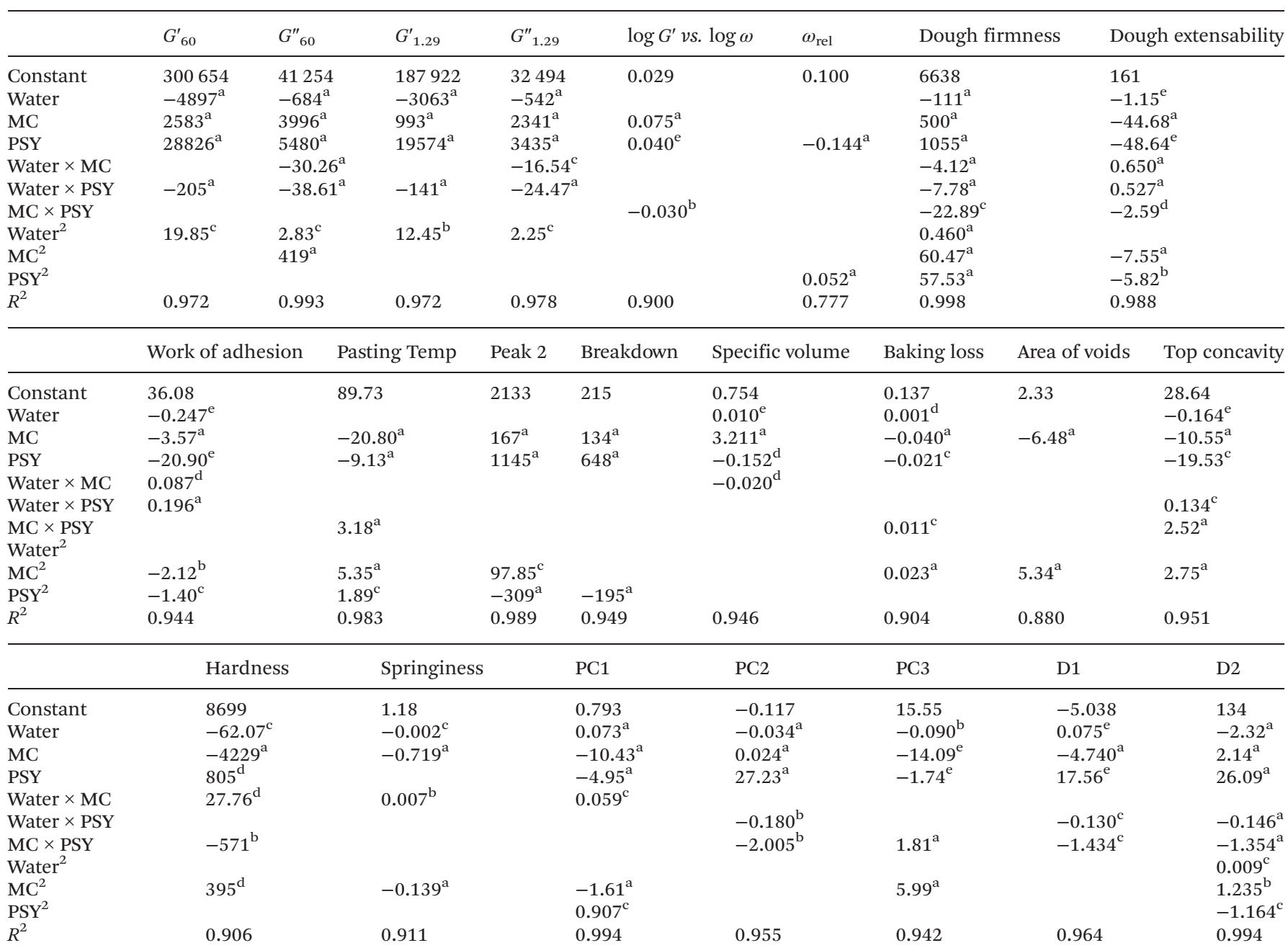

${ }^{\mathrm{a}} p<0.0001,{ }^{\mathrm{b}} p<0.001,{ }^{\mathrm{c}} p<0.01,{ }^{\mathrm{d}} p<0.05$ and ${ }^{\mathrm{e}} p>0.05$. 
$G^{\prime \prime}{ }_{60}, G^{\prime}{ }_{1.29}, G^{\prime \prime}{ }_{1.29}, \log G^{\prime} v s . \log \omega$, and $\omega_{\text {rel }}$ are listed. The correlation coefficients $\left(R^{2}\right)$ are higher than 0.9 for $G_{60}^{\prime}, G^{\prime \prime}{ }_{60}$, $\log G^{\prime}$ vs. $\log \omega$, and $R^{2}$ is higher than 0.75 for generalised Maxwell model parameters $\left(\omega_{\text {rel }}\right)$.

It can be seen that the addition of MC or PSY increased $G^{\prime}{ }_{60}, G^{\prime \prime}{ }_{60}, G^{\prime}{ }_{1.29}, G^{\prime \prime}{ }_{1.29}, \eta_{1.29}^{*}$, and $\eta_{60}^{*}$ which suggest a reinforcement effect and they also had positive contributions to the slope of $\log G^{\prime}$ versus $\log \omega$, which indicates less frequency dependence and more solid-like property. Water addition level is shown to had negative effects on $G_{60}^{\prime}, G^{\prime \prime}{ }_{60}, G_{1.29}^{\prime}, G^{\prime \prime}{ }_{1.29}$, $\eta_{1.29}^{*}$, and $\eta_{60}^{*}$ (insignificant influence on the slope of $\log G^{\prime}$ versus $\log \omega$ ). Increased water addition level also counteract the positive effect of PSY on $G^{\prime}$ as well as the positive effect of both PSY and MC on $G^{\prime \prime}$. These phenomena have been well documented as the results of the reinforcement effects of hydrocolloids on viscoelastic behaviours and the dilution effect of water on both flour and hydrocolloids, respectively. ${ }^{8,18,20}$ Additionally, as shown in S1 (ESI $\dagger$ ), comparing the first column (a), flour particles are distributed more evenly with the addition of PSY or MC, although there is no significant difference between MC containing (DA) doughs and PSY containing (DP) doughs. The reason might be that higher viscosity increased the effectiveness of shearing during dough mixing. This more homogeneous packing pattern could also contribute to the reinforcement effect. At a low water addition level of $110 \mathrm{~g}$ per $100 \mathrm{~g}$ flour, the influence of PSY on $G^{\prime}{ }_{60}$, which increased it by 6.5 times from $2200 \mathrm{~Pa}$ to $14700 \mathrm{~Pa}$, is more significant than MC (increased $G^{\prime}{ }_{60}$ by 3.3 times). This is also seen in the regression model of $G_{1.29}^{\prime}$. However, MC is more influential on $G^{\prime \prime}{ }_{60}$ (increased by 10 to 20 times depending on water content) while PSY is more influential on $G^{\prime \prime}{ }_{1.29}$ (increased by 6 to 8 times). MC is known to significantly increase viscosity when dissolved in water and the mechanical spectrum show higher $G^{\prime}$ at high frequency and higher $G^{\prime \prime}$ at low frequency. ${ }^{37}$ In contrast, PSY shows gel-like property. ${ }^{14,38-41}$ Therefore, MC and PSY showed different contributions to the elastic and viscous moduli of the doughs at different frequencies. The difference between the rheological properties of MC and PSY and their different water-binding abilities might be the reasons for their differences in the counteracting effect of water. Interestingly, MC $\times$ PSY had a negative coefficient in the model of $\log G^{\prime} v s$. $\log \omega$, which indicates a negative contribution. It might be similar to the depletion effects on $G^{\prime}$ between two types of HPMC due to, as proposed, water competition. ${ }^{20}$

Relaxation frequency $\left(\omega_{\text {rel }}\right)$ was predicted based on the generalised Maxwell model and, as seen in Table 3, PSY is the only term having a significant $(p<0.0001)$ effect on $\omega_{\text {rel }}$ with satisfactory fitting $\left(R^{2}=0.777\right)$ where $\omega_{\text {rel }}$ was significantly decreased when PSY addition increased to $1 \mathrm{~g}$ per $100 \mathrm{~g}$ flour but was less influenced with further additions. The relaxation of gluten free doughs in this study is of the order of 0.01 to 0.1 rad $\mathrm{s}^{-1}$ which is much higher than wheat doughs which have a relaxation frequency of the order of $10^{-4} \mathrm{rad} \mathrm{s}^{-1} \cdot{ }^{42,43}$ It suggests that gluten free doughs are more fluid-like at the deformation rates during proving compared to wheat doughs.
The relaxation frequency is also higher than the strain rate $\left(10^{-4}\right.$ to $10^{-3} \mathrm{~s}^{-1}$ as reported by Babin et al. ${ }^{33}$ and Grenier et $a l .{ }^{34}$ ) during proving which indicates that the doughs behave like fluids during proving. However, the deformation rate can be influenced by the relaxation time, in addition to the stress caused by the generation of $\mathrm{CO}_{2}$. It is worth conducting further experiments to study the correlations between relaxation time and deformation rate during proving.

\subsection{Empirical rheological analysis of dough properties}

Large deformation rheological properties of gluten free doughs were tested by backward extrusion experiments and the dough stickiness was measured by an SMS/ChenHoseney Dough Stickiness rig. The coefficients in the regression models for dough firmness, extensibility, and work of adhesion are listed in Table 3. The influences on dough firmness (Table 3) and consistency (data not shown) are generally consistent with the influences on the fundamental rheological parameters in that (1) water had negative effects while MC and PSY had positive effects, which can be counteracted by higher water addition levels, (2) the interaction MC $\times$ PSY had a negative contribution. The contributions of different model terms to the different aspects of dough rheological properties (such as $G^{\prime}, G^{\prime \prime}$, and $\log G^{\prime} v s$. $\log \omega$ ) can be differentiated in the fundamental rheological measurements. However, all single, interacting, and quadratic model terms contribute to dough firmness and consistency by empirical measurements.

High extensibility and work of adhesion describe a homogenous and smooth dough with high consistency which has a good performance in extensional flow. Additional water decreased the extensibility by a factor of 3 from $34.5 \mathrm{~mm}$ at $110 \mathrm{~g}$ of water to $11.5 \mathrm{~mm}$ with $130 \mathrm{~g}$ of water. Adding hydrocolloids eliminated the effect of additional water on dough extensibility. Adding MC resulted in extensibility of around $60 \mathrm{~mm}$ irrespective of the water content. PSY increased the extensibility to $29 \mathrm{~mm}$ with $130 \mathrm{~g}$ of water while the highest value $(38 \mathrm{~mm})$ can be obtained with $110 \mathrm{~g}$ of water and $0.8 \mathrm{~g}$ of PSY. These are similar to the parameters in the model for the work of adhesion. It suggests that the high extensibility and work of adhesion are achieved by concurrently increased additions of water and hydrocolloids, especially MC.

\subsection{Pasting properties of flour blends}

Pasting properties measured by RVA describe gelatinisation and retrogradation of starch and flour. A typical pasting curve of rice flour (run 11) is shown in Fig. 2a. Run 15, run 4, and run 16 containing different amount of MC and/or PSY showed lower pasting temperatures which appear as a shoulder or small peak (peak 1). Interestingly, an additional peak (peak 3) was observed on the viscosity profiles of PSY-containing samples. The viscosity profiles of run 15, run 13, run 2 and run 9 are shown in Fig. 2b, which contain flour, MC, and different amount of PSY. As the PSY content increased, peak 3 became more pronounced which indicates that peak 3 is caused by and increases with the PSY addition. Due to the 

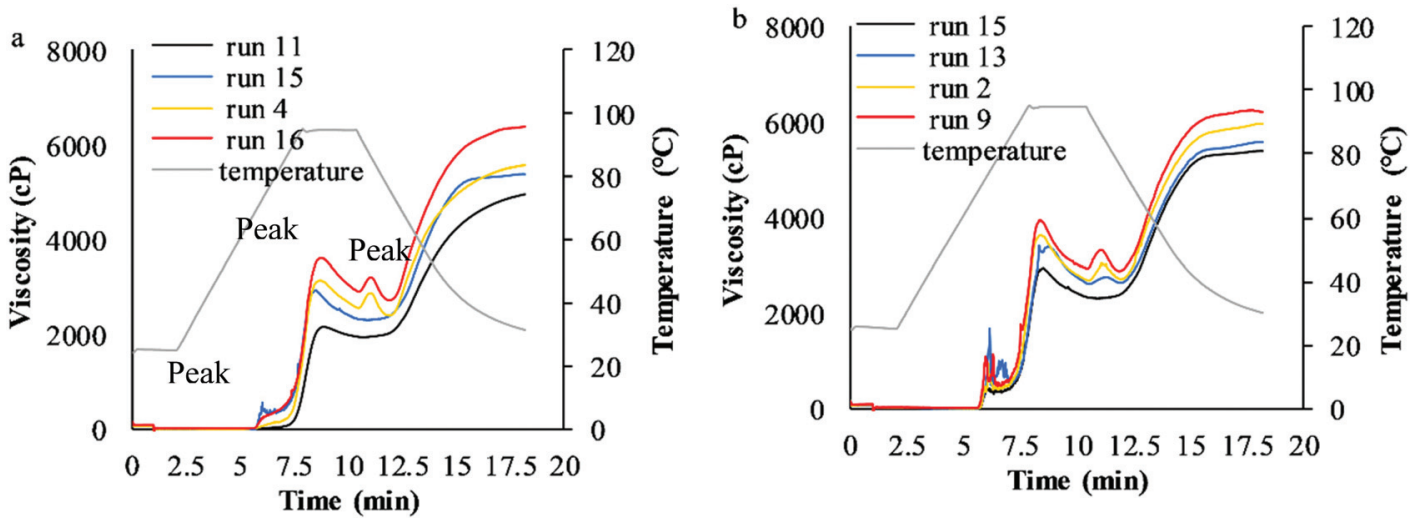

Fig. 2 Pasting profiles of flour blends containing various amounts of hydrocolloids (a) or containing a constant level of MC and increasing amounts of PSY (b). The flour/hydrocolloids ratios are shown in Table 1 that run 11 contains rice flour only; run 15 contains $2.5 \mathrm{~g}$ flour and $0.05 \mathrm{~g}$ MC; run 4 contains $2.5 \mathrm{~g}$ flour and $0.05 \mathrm{~g}$ PSY; run 16 contains $2.5 \mathrm{~g}$ flour, $0.0375 \mathrm{~g} \mathrm{MC}$ and $0.05 \mathrm{~g}$ PSY; run 13 contains $2.5 \mathrm{~g}$ flour, $0.05 \mathrm{~g}$ MC and $0.0125 \mathrm{~g}$ PSY; run 2 contains $2.5 \mathrm{~g}$ flour, $0.05 \mathrm{~g} \mathrm{MC}$ and $0.025 \mathrm{~g}$ PSY; run 9 contains $2.5 \mathrm{~g}$ flour, $0.05 \mathrm{~g}$ MC and $0.0375 \mathrm{~g}$ PSY.

existence of the extra peak compared to the classic analysis, extra parameters were applied to describe the pasting properties of gluten free flour blends which are peak 2, peak 3, trough 1 , trough 2 , trough total, breakdown, final viscosity, setback, peak 2 time, peak 3 time, peak 2 temp, peak 3 temp, and pasting temp.

As described by the model equation (Table 3) for pasting temperature, adding MC up to $2 \mathrm{~g}$ per $100 \mathrm{~g}$ of flour resulted in a decrease of pasting temperature from 90 to $70{ }^{\circ} \mathrm{C}$ while PSY was less influential and decreased the pasting temperature to $80{ }^{\circ} \mathrm{C}$. It has been concluded that the reduction of pasting temperature is common for most starch-hydrocolloid mixtures including the mixtures of $\mathrm{MC}$ and wheat flour and mixtures of MC and maize starch. ${ }^{44-46}$ The viscosity-increasing effect of MC allows the detection of earlier granule swelling. ${ }^{47}$ Sullo and Foster ${ }^{45}$ suggested that the recording of lower pasting temperature might due to the viscosity increase caused by the thermal gelation of MC which occurs at a lower temperature than the significant swelling of starch granules, which enhances starch granule interactions by increasing starch concentration. Naruenartwongsakul et $a l^{46}$ suggested that the concentration increase of starch is due to water competition with MC. Therefore, it can be concluded that the decreased pasting temperature is a result of water and volume competition between starch and MC. This could also be the reason for the influence of PSY on the reduction of pasting temperature. It is worth mentioning that pasting temperature is identified by the software when the viscosity-increasing rate reaches a predefined value. A low value might lead to significantly different pasting temperatures. However, the interaction $\mathrm{MC} \times$ PSY has a positive term coefficient which indicates that the increase of addition level of one hydrocolloid negates the decreasing effect of the other hydrocolloid on pasting temperature. The reason might be the same as the negative contribution to $\log G^{\prime} v s$. $\log \omega$ as mentioned in the previous section that water competition between MC and PSY leads to depletion between them.
As for the main viscosity peak (peak 2), MC positively contributed to this response and increased it from $2200 \mathrm{cP}$ to $2860 \mathrm{cP}$ at $2 \mathrm{~g}$ of MC. The effect of PSY on peak 2 was more pronounced than MC. Peak 2 increased to $3150 \mathrm{cP}$ when PSY increased to $1.5 \mathrm{~g}$ per $100 \mathrm{~g}$ flour but it was increased to a lesser extent by the further PSY addition. Similar contributions of MC and PSY to trough (data not shown) were also observed. There is no interacting effect of MC $\times$ PSY on the responses at high temperatures. MC gels at high temperature, which forms gel particles under the shearing condition in RVA and significantly increases the viscosity. ${ }^{45}$ In contrast, PSY melts at high temperature ${ }^{41}$ hence, it would be expected to be less influential on viscosity which is contrary to what was observed. Norton et al..$^{48}$ and Norton et al. ${ }^{49}$ illustrated the formation of fluid gels taking agar as the example which gels during cooling. The fluid gels were prepared by cooling agar with shearing which started with the formation of gel nuclei, which grew in size and induced the increase of viscosity. Below a certain temperature, the formation of particle nuclei ended and further molecular ordering occurred within the formed particles accompanied by reinforcement of the particles. The particles were hairy due to the disordered chains, which became smoother due to further molecular ordering which was accompanied by a decrease in viscosity. As PSY restructures during cooling and self-association has been observed, ${ }^{50,51}$ it might undergo a similar process as the formation of agar fluid gel with shearing in the RVA conditions. In the shearing field at the high temperature corresponding to the temperature range from peak 2 to trough, PSY associates and forms hairy weak gel particles, which interact with other particles or breakdown into smaller fragments. Therefore, PSY contributes more to viscosity than MC, which forms elastic, stable, and smooth particles. The limited contribution to peak 2 of PSY at a high addition level $(>1.5 \mathrm{~g})$ might due to a more extensive self-association when the concentration increased which did not sufficiently contribute to the viscosity. 
The unique peak 3 due to PSY occurred at approximately $85{ }^{\circ} \mathrm{C}$ in a similar temperature range to the trough. It is suggested by James M. Cowley (personal communication) that peak 3 is attributed to the formation of a complex with amylose as PSY becomes compatible with the interaction with amylose at this temperature. It is also possibly due to the selfassociation of PSY including the formation of hairy PSY gel particles, reinforcement of these particles by interior structuring, and interactions between the hairy particles which contribute to the increase of viscosity, and superficial ordering of the hairy particles which causes a decrease of viscosity. Hence, a balance between the formation of weak PSY gel particles, PSY particle interactions, PSY particle breakdown, and interaction with amylose is critical for the pasting behaviour at this temperature range.

According to the model in Table 3 , the addition of MC in the tested range (from 0 to $2 \mathrm{~g}$ ) increased the breakdown, which describes the loss of integrity of starch granules, by 268 $\mathrm{cP}$ from $215 \mathrm{cP}$, but PSY increased it by $605 \mathrm{cP}$ with $1.6 \mathrm{~g}$ of PSY. This phenomenon has been interpreted as a result of the viscosity increase by hydrocolloid addition which leads to an increase of shear force exerted on starch granules. ${ }^{46,52}$ However, it can also reflect the viscosity changes of hydrocolloids. As discussed above, in the temperature range from peak 2 to trough, PSY may undergo changes from softer but hairy particles, which interact with each other, to rigid but and smooth particles with less interaction, as well as possible temporary interaction with amylose. These phenomena contribute to a more significant increase in breakdown value. The limited increase of breakdown by PSY with addition levels higher than $1.6 \mathrm{~g}$ is correlated to the influence of PSY on peak 2 .

\subsection{Bread qualities}

Specific volume is one of the most important parameters describing bread quality. As shown in Table 3, MC had the most significant $(p<0.0001)$ positive contribution to the specific volume. It doubled specific volume when its addition level increased from 0 to $2 \mathrm{~g}$. As shown in $\mathrm{S} 2 \uparrow$ column $\mathrm{b}, \mathrm{MC}$ appears more continuous which is similar to gluten, at least in terms of the microstructural distribution. The similarity in structure might suggest a similarity in functionalities between MC and gluten that both of them form a continuous phase in addition to the starch matrix to stabilise the loaf structure and be beneficial to a higher loaf volume. In addition, as shown in $\mathrm{S} 1 \dagger \mathrm{DA}$ column $\mathrm{b}$ that MC appears as discrete particles instead of a homogeneous solution, the thermal gel of $\mathrm{MC}$ at the higher temperature might be limited due to limited hydration of MC powder as a result of the water content in the formulation and water competition between flour, PSY and MC. Therefore, the mobility of MC molecules might be reduced which restrains them from forming junction zones and inhibits the thermal gelation process. Therefore, it is more likely that, even at high temperature during baking, MC contributes to the loaf stability in a manner of increasing the viscous part of the viscoelasticity instead of the elastic part. This behaviour allows for the further expansion of gas cells during baking.
PSY is slightly detrimental to specific volume. The detrimental effect of PSY on specific volume was also confirmed by Mancebo et al. ${ }^{18}$ When MC addition was higher than $0.5 \mathrm{~g}$, additional water started to decrease specific volume. This is contrary to what has been reported by others that a higher water addition level contributes to a higher specific volume. ${ }^{17,53}$ Hence, the influences of hydrocolloids and water on loaf volume can be complicated. The contrary observation in this study might due to different water absorbabilities of ingredients and, in a certain addition range, water might over dilute the doughs which lead to lower stability, poorer gas trapping ability, and lower loaf volume.

However, volume increase may be accompanied by large voids in the crumb structure. It can be seen that MC and $\mathrm{MC}^{2}$ have the most significant influence on the area of voids $(p<$ $0.0001)$. The area of voids significantly increased by 10 fold from $1.3 \%$ at $1 \mathrm{~g}$ of MC to $10.7 \%$ with $2 \mathrm{~g}$ of MC. The existence of voids in gluten free bread has been also observed by Haque and Morris, ${ }^{7}$ McCarthy et al. ${ }^{17}$ and Nishita et al. ${ }^{6}$ due to excessive water addition which leads to overexpansion and dough instability. However, the interacting influences of hydrocolloids and water on the area of voids can be complicated. Overexpansion and dough instability might explain the formation of voids in the loaves made with high water and hydrocolloid contents. High water content dilutes the flour or starch content which leads to weaker walls of gas cells and promotes coalescence especially during proving and early stage of baking. This was also thought to occur by McCarthy et al. ${ }^{17}$ Starch gelatinisation is one of the dominant transitions in the formation of bread crumb structure. ${ }^{54}$ Therefore, dilution of starch also leads to weaker crumb structure which is less tolerant of the compression and expansion, as described by Wagner et al. ${ }^{55}$ during baking.

In contrast, formulations with lower water content and higher hydrocolloid contents lead to a high viscosity of doughs and air pockets can be trapped during dough mixing and loading into the pans. The size of voids might then be promoted by Ostwald ripening or further coalescence of small gas cells. Based on their appearance and formation reasons, the voids can be classified into three types (S3 $\dagger$ ). Two types of voids have smooth inner surfaces and the other has a coarse surface. Type 1 with a smooth surface might be caused by air trapped during mixing. Type 2 with a smooth surface was formed with excessive water addition. It is characterised by moving traces of gas cells which can, sometimes, form cluster of smooth tunnels. The formation of type 1 and type 2 can include Ostwald ripening and coalescence of gas cells during proving and early stage of baking before starch gelatinisation. Type 1 voids are sometimes big and impermeable, leading to shrinkage after baking which is also reported by Purna et al. ${ }^{56}$ Type 3 voids have a coarse inner surface. The possible reason is high pressure during baking after starch gelatinisation is finished and sponge crumb structure is formed, which rips the porous structure.

Another parameter representing dough and crumb instability is top concavity, which was obtained from the C-cell evalu- 
ation describing the concavity on the top of the loaves. It is caused by collapse during proving or the early stages of baking. Both the addition of MC and PSY reduced top concavity. MC resulted in a decrease of top concavity from $10.6 \%$ to $0.48 \%$ at $2 \mathrm{~g}$ addition level while PSY was less efficient and resulted in a top concavity of $1 \%$.

Textural properties are another type of descriptive parameter of bread quality. According to preliminary tests, the gas cells can occupy up to $60 \%$ of the loaf volume. Therefore, the loaves were compressed by $65 \%$ of their original height in TPA so the properties of bulk crumb were also recorded in addition to the properties of the walls of gas cells. In addition, a higher strain mimics the oral process. According to the model, PSY significantly increased the crumb hardness from $1500 \mathrm{~g}$ to $3000 \mathrm{~g}$ with $110 \mathrm{~g}$ of water and from $750 \mathrm{~g}$ to $2300 \mathrm{~g}$ with $130 \mathrm{~g}$ of water. This crumb hardening effect of PSY is in agreement with Mancebo et al. ${ }^{18}$ Hardness decreased from $1500 \mathrm{~g}$ to $700 \mathrm{~g}$ with the water addition increased from $110 \mathrm{~g}$ to $130 \mathrm{~g}$. However, when $2 \mathrm{~g}$ of MC was incorporated, the variation of water and PSY only caused $500 \mathrm{~g}$ difference in hardness. The reinforcement effects of PSY and dilution effect of water influenced the strength of the crumb cell walls and, therefore, contributed to their positive and negative effect on hardness respectively. As shown in S2, $\uparrow$ PSY exists as discrete particles in the cooked flour/hydrocolloid gels. Combining with the "weak gel' property of PSY, ${ }^{7}$ PSY can be expected to increase the strength of starch gels which compose the gas cell walls and, hence, increase the hardness of bread crumb. Their contributions to hardness are contrary to their contributions to specific volume as a denser crumb is harder. ${ }^{17}$ The addition of MC significantly increased specific volume and reduced crumb density and hardness. Therefore, the influence of PSY and water on the strength of gas cell walls was diminished.

As for springiness, MC had a quadratic influence on springiness and the addition level for the maximum springiness increased from 0.34 to $0.87 \mathrm{~g}$ per $100 \mathrm{~g}$ flour with the increase of water addition in the tested range (110 to $130 \mathrm{~g}$ per $100 \mathrm{~g}$ flour). Surprisingly, the influence of PSY was insignificant. Higher springiness with PSY addition was observed in our preliminary experiments where a different rice flour was used and samples were compressed to a different degree. High springiness by PSY addition was also reported by Haque and Morris. ${ }^{7}$ It is likely that the influence of PSY on the springiness of gluten free bread is dependent on the properties of the flour/starch. Additionally, gluten free bread is less recoverable (low springiness), especially under high compression.

\subsection{Simultaneous application of principal component analysis and response surface method}

Response surface methodology has been applied to investigate the effects of different factors on gluten free doughs and bread, to optimise formulation and process. ${ }^{17,20,57-60}$ However, the number of responses that can be analysed is limited as they are analysed individually but the gluten free bread and doughs cannot be fully described by the limited amount of responses. Therefore, principal component analysis (PCA) was applied to reduce the number of response dimensions. In this study, 74 responses were generated and analysed by PCA, where principal components with different eigenvalues were obtained. The first three principal components dominated with the eigenvalues of $31.53,17.15$, and 9.22 representing $41.5 \%, 22.6 \%$ and $12.1 \%$ of the variance in the data set. They were extracted as new responses in RSD analysis and the models are listed in Table 3. The loadings of the original 74 responses on PC1, PC2, and PC3 are plotted in Fig. 3a and b.

As shown in Fig. 3a, the specific volume is positively correlated with proofed loaf volume and it is closer to the final proving volume (volume increase final) instead of the maximum volume (volume increase max), which suggests that the volume after proving is more critical in deciding the baked loaf volume. Specific volume is clustered with many C-cell parameters including the ones describing the size of bread slices, crumb structure, and the volume of voids which is discussed in section 3.4. Specific volume is negatively related to springiness, hardness, chewiness, and gumminess. A similar relationship was also reported by Gallagher et $a l^{53}$ and Scanlon and Zghal. ${ }^{61}$ As for the relationship between specific volume and dough rheological properties, specific volume is closely and positively correlated with extensibility followed by work of adhesion, measured by empirical rheological tests. It is also slightly close to $\tan \delta$, followed by $\log G^{\prime} v s . \log \omega$ and $\log \eta^{*} v s . \log \omega$ measured by fundamental rheological tests.

Although PC1 is mainly loaded by parameters characterising bread qualities and pasting profiles, specific volume and correlated responses had high loadings on both PC1 and PC2 and they laid close to the diagonal of the PC1-PC2 plot. Therefore, new coordinate axes (D1 and D2, as shown in Fig. 3a) were generated by rotating the original PC1-PC2 coordinates by $45^{\circ}$. The application of diagonal axes has been adopted by Dewaest et al. ${ }^{62}$ The new axes were also analysed in RSD as new responses and listed in Table 3. It can be seen that MC, with a high term coefficient, had the most dramatic influence on PC1 than PSY and water. It decreased PC1 from 8.8 to -5.5 when $2 \mathrm{~g}$ of MC was added. As for D1, MC was the only influential term with a significance level of $p<0.0001$. Additionally, as discussed in previous sections, MC had significant effects on dough extensibility, specific volume, and crumb hardness. Therefore, it can be concluded that MC addition level is critical for a desirable dough. The dough with high extensibility and work of adhesion is expected to be homogeneous and smooth which has good performance in extensional flow. The gas cell wall undergoes extensional flow during gas cell growth. Therefore, these two responses can describe the dough quality in the development of the foam structure. Low extensibility and work of adhesion suggest that the doughs are rigid which prohibit the growth of gas cells. High extensibility and work of adhesion indicate that the gas cell walls allow the expansion of gas cells and be able to trap gas to a certain degree, which increases the loaf volume after proving and baking. Some parameters measured by fundamental rheological measurements, such as $\tan \delta$, also reflects flowability of doughs. However, the flowability of gas cell walls 


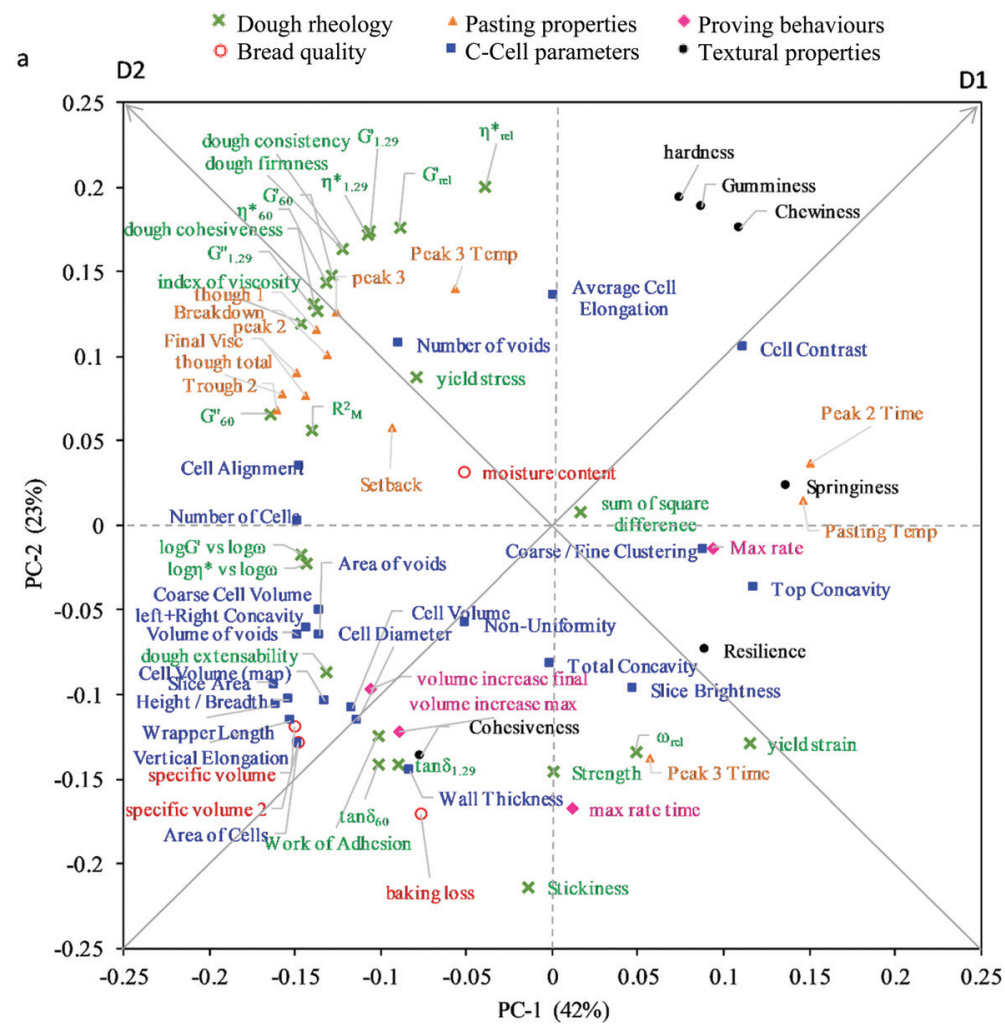

b

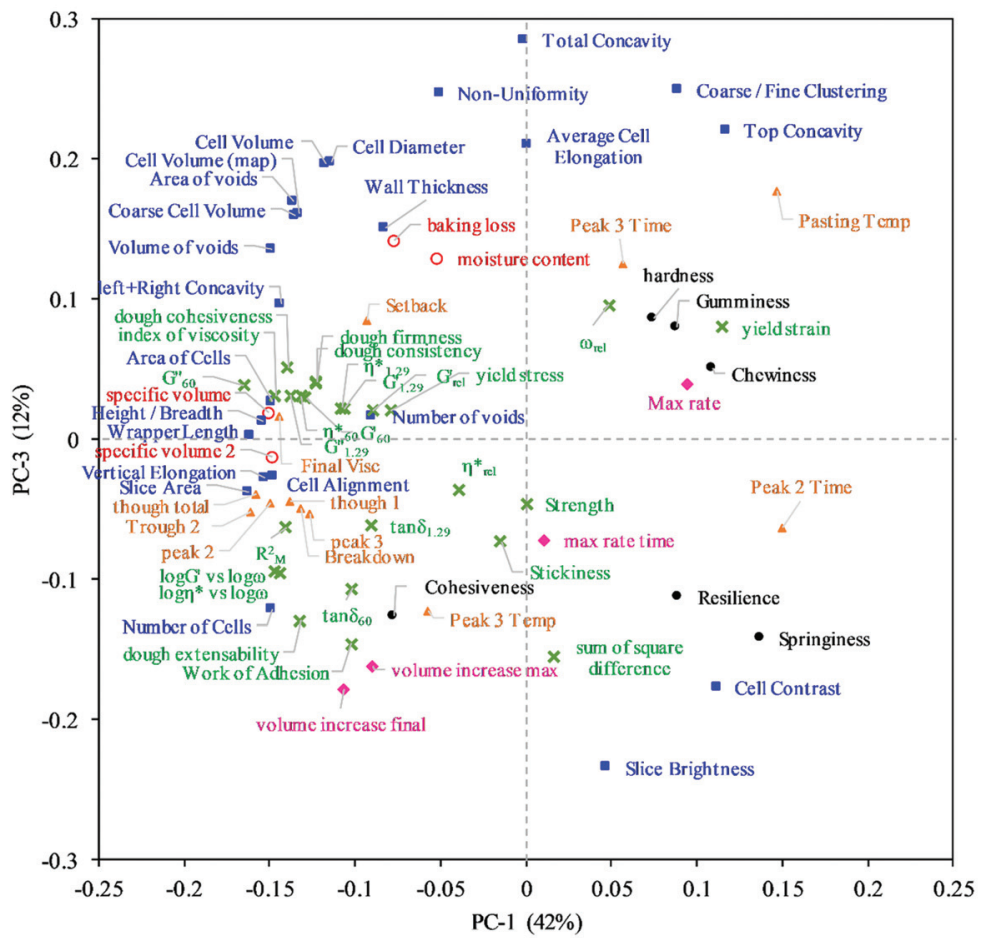

Fig. 3 Loading of responses on PC1 and PC2 (a) and on PC1 and PC3 (b). D1 and D2 are diagonal axes generated by rotating the original PC1-PC2 coordinates by $45^{\circ}$.

introduces instability and leads to the failure of the walls which lead to voids in crumb as discussed in section 3.4. A smaller loaf (low specific volume) will have a dense and tightly packed crumb structure with a hard crumb texture while a big loaf, in the contrast, has soft, tenuous but weak and less springy crumb. The term water $\times$ MC in the PC1 model 
suggests the interaction effects of these two factors on gluten free bread.

Other empirical rheological parameters, moduli measured by fundamental rheological tests, especially storage moduli, and most pasting properties are distributed more perpendicularly to specific volume in Fig. 3a indicating less significant correlations. Small amplitude oscillatory measurements can be considered as being inappropriate in the evaluation of application and processing scenarios of gluten free bread, as the deformation rate scales and experiment conditions do not simulate the conditions of dough making and baking. ${ }^{22}$ However, specific volume is clustered with these responses in the PC1-PC3 plotted (Fig. 3b) which suggests lower correlations between loaf volume and dough rheology.

It can be seen in Fig. 3a that most fundamental rheological parameters are close to dough firmness, cohesiveness, and consistency. Similar correlations were reported by Ronda et $a .^{20}$ although the geometry for empirical measurement was different. Therefore, the firmness or strength of gluten free doughs can be described by both large deformation empirical tests and small deformation fundamental tests. Relaxation frequency, along with dough strength and yield strain, is distributed opposite to the most other rheological responses and they are clustered with max rate time during proving and concavity of loaves. It suggests that these responses describe the proving behaviour, which influences the concavity of the loaves. These responses have high loadings on PC2 and are close to D2. According to the models listed in Table 3, PSY has the most significant influence on PC2 and D2 which increases PC2 from -3.8 to 10.8 and increases $\mathrm{D} 2$ from -8.7 to 6.8 . MC and water alone are less influential on PC2 and they increase or decreases D2 by approximately 2 respectively.

PC3 is mainly dependent on some bread quality parameters, empirical dough rheological parameters and volume increases during proving. Water is the only factor having a significant contribution among the three single terms (judged by 95\% confidence) indicating that water is influential in determining the residual information in the data set.

\section{Conclusion}

The study aimed at evaluating the influences of MC, PSY and water addition levels on gluten free doughs and bread, and the correlations between dough rheological properties, baking behaviours and bread qualities. Relaxation frequency of doughs was calculated by the generalised Maxwell model and it suggests that gluten free doughs are more fluid-like at the deformation rate during proving. The additions of both MC and PSY decreased pasting temperature due to volume and water competition. The addition of PSY has more significant influence than MC on pasting viscosity at high temperature (from peak to trough) and leads to an extra peak on the pasting profiles, which might rely on a balance between formation and breakdown of PSY weak gel particles, PSY particle interactions, and interaction with amylose. MC and PSY tend to have depletion effects, which lead to counteracted contributions to the frequency dependence of storage moduli, dough firmness, and pasting temperature.

Simultaneous application of PCA and RSD was successful to reduce the size of response dimensions and evaluate the correlations between responses. MC has a significant influence on dough extensibility and work of adhesion which evidenced to be good predictors of bread qualities including specific volume, crumb structure, and texture. Other dough rheological parameters including small amplitude oscillatory tests are more sensitive to the formulation variations, especially the PSY addition. They provide information such as dough stability and structure, describe proving behaviour, and related to loaf concavity. Combining PCA and RSD allows the analysis of a large amount of data which provides a relatively comprehensive characterisation of gluten free bread. Other parameters such as sensory evaluations could be involved in further investigations to provide a better understanding of gluten free bread.

\section{Conflicts of interest}

There are no conflicts of interest to declare.

\section{Acknowledgements}

This work was supported by the University of Nottingham (Vice-Chancellor's Scholarship for Research Excellence (International)) and Pepsico. The views and opinions expressed in this manuscript are those of the author and do not necessarily reflect the position or policy of Pepsico. The authors would also like to thank Khatija Nawaz Husain for her technical help with confocal laser scanning microscopy, which was conducted at the School of Life Sciences Imaging, and Microscopy Facility (SLIM) at the University of Nottingham.

\section{References}

1 C. Catassi, R. P. Anderson, I. D. Hill, S. Koletzko, E. Lionetti, N. Mouane, M. Schumann and S. K. Yachha, World perspective on celiac disease, J. Pediatr. Gastroenterol. Nutr., 2012, 55, 494-499.

2 G. Czaja-Bulsa, Non coeliac gluten sensitivity - A new disease with gluten intolerance, Clin. Nutr., 2015, 34, 189194.

3 T. Thompson, The nutritional quality of gluten-free foods, in Gluten-free food science and technology, ed. E. Gallagher, Wiley-Blackwell, Chichester, 2009, ch. 4, pp. 42-51.

$4 \mathrm{~J}$. Singh and K. Whelan, Limited availability and higher cost of gluten-free foods, J. Human Nutr. Diet., 2011, 24, 479-486.

5 R. S. Kadan, M. G. Robinson, D. P. Thibodeaux and A. B. Pepperman, Texture and other physicochemical properties of whole rice bread, J. Food Sci., 2001, 66, 940-944. 
6 K. D. Nishita, R. L. Roberts, M. M. Bean and B. M. Kennedy, Development of a yeast-leavened rice-bread formula, Cereal Chem., 1976, 53, 626-635.

7 A. Haque and E. R. Morris, Combined use of ispaghula and HPMC to replace or augment gluten in breadmaking, Food Res. Int., 1994, 27, 379-393.

8 A. Lazaridou, D. Duta, M. Papageorgiou, N. Belc and C. G. Biliaderis, Effects of hydrocolloids on dough rheology and bread quality parameters in gluten-free formulations, J. Food Eng., 2007, 79, 1033-1047.

9 E. Gallagher, T. R. Gormley and E. K. Arendt, Recent advances in the formulation of gluten-free cereal-based products, Trends Food Sci. Technol., 2004, 15, 143-152.

10 H. G. Masure, E. Fierens and J. A. Delcour, Current and forward looking experimental approaches in gluten-free bread making research, J. Cereal Sci., 2016, 67, 92-111.

11 M. R. Rao, D. U. Warrier, S. R. Gaikwad and P. M. Shevate, Phosphorylation of psyllium seed polysaccharide and its characterization, Int. J. Biol. Macromol., 2016, 85, 317-326.

12 J. W. Anderson, L. D. Allgood, A. Lawrence, L. A. Altringer, G. R. Jerdack, D. A. Hengehold and J. G. Morel, Cholesterol-lowering effects of psyllium intake adjunctive to diet therapy in men and women with hypercholesterolemia: meta-analysis of 8 controlled trials, Am. J. Clin. Nutr., 2000, 71, 472-479.

13 Y.-J. Song, M. Sawamura, K. Ikeda, S. Igawa and Y. Yamori, Soluble dietary fibre improves insulin sensitivity by increasing muscle glut-4 content in stroke-prone spontaneously hypertensive rats, Clin. Exp. Pharmacol. Physiol., 2000, 27, 41-45.

14 A. Haque, R. K. Richardson, E. R. Morris and I. C. M. Dea, Xanthan-like weak gel rheology from dispersions of ispaghula seed husk, Carbohydr. Polym., 1993, 22, 223-232.

15 C. Chieh, Water, in Bakery products science and technology, ed. Y. H. Hui, Blackwell Publishing Ltd, Oxford, 2006, ch. 7, pp. 211-232.

16 H. K. Leung, J. A. Magnuson and B. L. Bruinsma, Pulsed nuclear magnetic-resonance study of water mobility in flour doughs, J. Food Sci., 1979, 44, 1408-1411.

17 D. F. McCarthy, E. Gallagher, T. R. Gormley, T. J. Schober and E. K. Arendt, Application of response surface methodology in the development of gluten-free bread, Cereal Chem., 2005, 82, 609-615.

18 C. M. Mancebo, M. Á. San Miguel, M. M. Martínez and M. Gómez, Optimisation of rheological properties of gluten-free doughs with HPMC, psyllium and different levels of water, J. Cereal Sci., 2015, 61, 8-15.

19 N. O'Shea, C. Rößle, E. Arendt and E. Gallagher, Modelling the effects of orange pomace using response surface design for gluten-free bread baking, Food Chem., 2015, 166, 223230.

20 F. Ronda, S. Pérez-Quirce, A. Angioloni and C. Collar, Impact of viscous dietary fibres on the viscoelastic behaviour of gluten-free formulated rice doughs: A fundamental and empirical rheological approach, Food Hydrocolloids, 2013, 32, 252-262.
21 M. M. Martínez and M. Gómez, Rheological and microstructural evolution of the most common gluten-free flours and starches during bread fermentation and baking, J. Food Eng., 2017, 197, 78-86.

22 M. Mariotti, M. Lucisano, M. A. Pagani and P. K. W. Ng, The role of corn starch, amaranth flour, pea isolate, and psyllium flour on the rheological properties and the ultrastructure of gluten-free doughs, Food Res. Int., 2009, 42, 963-975.

23 A. Torbica, M. Hadnadev and T. Dapcevic, Rheological, textural and sensory properties of gluten-free bread formulations based on rice and buckwheat flour, Food Hydrocolloids, 2010, 24, 626-632.

24 A. Lazaridou and C. G. Biliaderis, Gluten-free doughs: Rheological properties, testing procedures - methods and potential problems, in Gluten-Free Food Science and Technology, ed. E. Gallagher, Wiley-Blackwell, Chichester, 2009 , ch. 5, pp. 52-82.

25 N. Bratchell, Multivariate response surface modelling by principal components analysis, J. Chemom., 1989, 3, 579588.

26 J. S. Ribeiro, R. F. Teofilo, F. Augusto and M. M. C. Ferreira, Simultaneous optimization of the microextraction of coffee volatiles using response surface methodology and principal component analysis, Chemom. Intell. Lab. Syst., 2010, 102, 45-52.

27 M. Das Purkayastha, G. Dutta, A. Barthakur and C. L. Mahanta, Tackling correlated responses during process optimisation of rapeseed meal protein extraction, Food Chem., 2015, 170, 62-73.

28 Y. Ren, B. R. Linter and T. J. Foster, Starch replacement in gluten free bread by cellulose and fibrillated cellulose, Food Hydrocolloids, 2020, 107, 105957.

29 M. Baumgaertel and H. H. Winter, Interrelation between continuous and discrete relaxation time spectra, J. NonNewtonian Fluid Mech., 1992, 44, 15-36.

30 J. D. Ferry, Viscoelastic properties of polymers, John Wiley \& Sons, New York, 3rd edn, 1980.

31 H. M. Laun, Prediction of elastic strains of polymer melts in shear and elongation, J. Rheol., 1986, 30, 459-501.

32 W. Z. Chen and R. C. Hoseney, Development of an objective method for dough stickiness, LWT-Food Sci. Technol., 1995, 28, 467-473.

33 P. Babin, G. Della Valle, H. Chiron, P. Cloetens, J. Hoszowska, P. Pernot, A. L. Réguerre, L. Salvo and R. Dendievel, Fast X-ray tomography analysis of bubble growth and foam setting during breadmaking, J. Cereal Sci., 2006, 43, 393-397.

34 D. Grenier, T. Lucas and D. Le Ray, Measurement of local pressure during proving of bread dough sticks: Contribution of surface tension and dough viscosity to gas pressure in bubbles, J. Cereal Sci., 2010, 52, 373-377.

35 H. M. Wyss, K. Miyazaki, J. Mattsson, Z. B. Hu, D. R. Reichman and D. A. Weitz, Strain-rate frequency superposition: A rheological probe of structural relaxation in soft materials, Phys. Rev. Lett., 2007, 98, 238303. 
36 B. M. Erwin, S. A. Rogers, M. Cloitre and D. Vlassopoulos, Examining the validity of strain-rate frequency superposition when measuring the linear viscoelastic properties of soft materials, J. Rheol., 2010, 54, 187-195.

37 A. Haque, R. K. Richardson, E. R. Morris, M. J. Gidley and D. C. Caswell, Thermogelation of methylcellulose .2. Effect of hydroxypropyl substituents, Carbohydr. Polym., 1993, 22, 175-186.

38 Q. Guo, S. W. Cui, Q. Wang, H. D. Goff and A. Smith, Microstructure and rheological properties of psyllium spolysaccharide gel, Food Hydrocolloids, 2009, 23, 15421547.

39 A. Farahnaky, H. Askari, M. Majzoobi and G. Mesbahi, The impact of concentration, temperature and $\mathrm{pH}$ on dynamic rheology of psyllium gels, J. Food Eng., 2010, 100, 294-301.

40 L. Yu, G. E. Yakubov, W. Zeng, X. Xing, J. Stenson, V. Bulone and J. R. Stokes, Multi-layer mucilage of Plantago ovata seeds: Rheological differences arise from variations in arabinoxylan side chains, Carbohydr. Polym., 2017, 165, 132-141.

41 Y. Ren, G. E. Yakubov, B. R. Linter, W. MacNaughtan and T. J. Foster, Temperature fractionation, physicochemical and rheological analysis of psyllium seed husk heteroxylan, Food Hydrocolloids, 2020, 104, 105737.

42 M. Meerts, R. Cardinaels, F. Oosterlinck, C. M. Courtin and P. Moldenaers, The interplay between the main flour constituents in the rheological behaviour of wheat flour dough, Food Bioprocess Technol., 2017, 10, 249-265.

$43 \mathrm{~J}$. Lefebvre, An outline of the non-linear viscoelastic behaviour of wheat flour dough in shear, Rheol. Acta, 2006, 45, 525-538.

44 J. N. BeMiller, Pasting, paste, and gel properties of starchhydrocolloid combinations, Carbohydr. Polym., 2011, 86, 386-423.

45 A. Sullo and T. J. Foster, Characterisation of starch/cellulose blends, Annu. Trans. - Nord. Rheol. Soc., 2010, 18, 1-7.

46 S. Naruenartwongsakul, M. S. Chinnan, S. Bhumiratana and T. Yoovidhya, Pasting characteristics of wheat flourbased batters containing cellulose ethers, LWT-Food Sci. Technol., 2004, 37, 489-495.

47 J. Blanshard, Starch granule structure and function: a physicochemical approach, in Starch: Properties and Potential, ed. T. Galliard, John Wiley and Sons, New York, 1987, pp. 16-54.

48 I. T. Norton, T. J. Foster and C. R. T. Brown, in Gums and stabilisers for the food industry, ed. P. A. Williams and G. O. Phillips, Royal Society of Chemistry, Cambridge, 1998, ch. 4, vol. 9, pp. 259-268.
49 I. T. Norton, D. A. Jarvis and T. J. Foster, A molecular model for the formation and properties of fluid gels, Int. J. Biol. Macromol., 1999, 26, 255-261.

50 L. Yu, G. E. Yakubov, M. Martínez-Sanz, E. P. Gilbert and J. R. Stokes, Rheological and structural properties of complex arabinoxylans from Plantago ovata seed mucilage under non-gelled conditions, Carbohydr. Polym., 2018, 193, 179-188.

51 Y. Ren, B. R. Linter and T. J. Foster, Cellulose fibrillation and interaction with psyllium seed husk heteroxylan, Food Hydrocolloids, 2020, 104, 105725.

52 D. D. Christianson, Gelatinization of wheat starch as modified by xanthan gum, guar gum, and cellulose gum, Cereal Chem., 1981, 58, 513-517.

53 E. Gallagher, T. R. Gormley and E. K. Arendt, Crust and crumb characteristics of gluten free breads, J. Food Eng., 2003, 56, 153-161.

54 W. Zhou and N. Therdthai, Three-dimensional CFD modeling of a continuous industrial baking process, in Computational fluid dynamics in food processing, ed. D. Sun, CRC Press, Boca Raton, 2007, ch. 9, pp. 306-331.

55 M. Wagner, S. Quellec, G. Trystram and T. Lucas, MRI evaluation of local expansion in bread crumb during baking, J. Cereal Sci., 2008, 48, 213-223.

56 S. K. G. Purna, R. A. Miller, P. A. Seib, R. A. Graybosch and Y. C. Shi, Volume, texture, and molecular mechanism behind the collapse of bread made with different levels of hard waxy wheat flours, J. Cereal Sci., 2011, 54, 37-43.

57 C. Marco and C. M. Rosell, Functional and rheological properties of protein enriched gluten free composite flours, J. Food Eng., 2008, 88, 94-103.

58 N. O'Shea, C. Rossle, E. Arendt and E. Gallagher, Modelling the effects of orange pomace using response surface design for gluten-free bread baking, Food Chem., 2015, 166, 223-230.

59 H. D. Sanchez, C. A. Osella and M. A. de la Torre, Optimization of gluten-free bread prepared from cornstarch, rice flour, and cassava starch, J. Food Sci., 2002, 67, 416-419.

60 C. Collar, P. Andreu, J. C. Martinez and E. Armero, Optimization of hydrocolloid addition to improve wheat bread dough functionality: a response surface methodology study, Food Hydrocolloids, 1999, 13, 467-475.

61 M. G. Scanlon and M. C. Zghal, Bread properties and crumb structure, Food Res. Int., 2001, 34, 841-864.

62 M. Dewaest, C. Villemejane, S. Berland, S. Neron, J. Clement, A. Verel and C. Michon, Effect of crumb cellular structure characterized by image analysis on cake softness, J. Texture Stud., 2018, 49, 328-338. 